\title{
GENETIC STUDIES ON THE PHOTOTHERMO SENSITIVE GENIC MALE STERILITY (PTGMS) AND ITS UTILIZATION IN RICE BREEDING. \\ El-Diasty, Z. M. ${ }^{1}$; H. F. El-Mowafi ${ }^{2}$; M. S. Hammada ${ }^{3}$ and R.M. Abdallah ${ }^{2}$ \\ 1) Genetic Dept., faculty of Agric., Mansoura University, Egypt. \\ 2) Rice Res. and Training Center (RRTC), Sakha, kafr El-Sheikh, Egypt. \\ 3) Genetic Dept., faculty of Agric.,Domiata Mansoura University, Egypt.
}

\section{ABSTRACT}

Studies of heterosis and combining ability of six photo /thermo-sensitive testers were estimated using line $\mathrm{x}$ tester analysis for some agronomic characters and yield and its components to get useful information for two-line system in hybrid rice breeding in Egypt .

The outstanding hybrid combinations for grain yield plant $^{-1}$ were PTGMS$5 /$ Dular $(45.26 \%)$ with significant standard heterosis for ,panicle length $(28.22 \%)$ productive tillers plant $^{-1}(24.19 \%)$,panicle weight $(26.93 \%)$, filled grains panicle ${ }^{-1}$ $(25.89 \%)$,spikelets panicle ${ }^{-1}(14.19 \%)$,spikelet fertility $\%(10.20 \%)$ and 100 -grain weight $(35.62 \%)$.In the mean time ,PTGMS-14/Norin PL9,PTGM-7/Dular ,PTGMS14/Giza178 and PTGMS-5/Giza 178 were considered as promising hybrids.

Among the six PTGMS lines,PTGMS-5 and PTGMS-14 were the best general combiners for grain yield and all studies characters.The testers, Dular, Giza178,M202 and Pecos were the best general combiners among testers for grain yield and most studies characters.

\section{INTRODUCTION}

Rice (Oryza sativa, L.) is the main food for about half of the world population. Indica type rice varieties feed about 3 billion people, predominantly in developing contries. Rice provides $23 \%$ of global human per capita energy and $16 \%$ per capita protein. Rice protein ranks high in nutritional quality among cereals. Rice also provides minerals, vitamins, and fiber (Alam et al., 1998). The rapidly increasing demand for rice and the continuous decrease in rice growing areas have resulted in search to improve rice production. In addition to improved varieties and new plant type, the use of hybrid rice may provide the target requirement of $15 \mathrm{t} / \mathrm{ha}$.

Hybrid rice has proven to be an effective and economical way to increase rice production output. It is easy to obtain about $15-20 \%$ higher yield just growing hybrid rice instead of the common varieties .

Two-line system based on photo/thermo-sensitive genic male sterile (P/TGMS) lines could be used. Since the gene(s) expression of P/TGMS is controlled by environment, the P/TGMS also called environmental genic male sterile (EGMS). The EGMS lines are sterile in long day (LD) and / or high temperature (HT) and become fertile in short day (SD) and / or low temperature (LT). During the sterile phase they can be used to produce hybrid seeds by crossing them with normal fertile male line,while in fertile phase they propagate themselves. The EGMS lines possess the following advantages: (1) Higher selection and utilization; (2) Not sensitive to cytoplasmic type and sterile lines having different cytoplasmic types can be 
bred easily; (3) Resources of restoring genes are wide ranging which can be used as its restoring lines provided they are not the heterozygotes of this recessive genic male sterile gene, thus up to date achievements in research of genetic breeding of rice can be collected and utilized for exploitation of heterosis.

\section{MATERIALS AND METHODS}

The experiment comprised progenies derived from 50 cross combinations generated through line $x$ tester mating design. Six Photoperiod/Thermo-sensitive genic male sterile ( PTGMS) Viz; PTGMS-1, 2, 4, 5, 7 and PTGMS-14 developed by Egyptian Hybrid Rice Breeding Program in Egypt were used as female lines. Seven diverse tester varieties Viz., Pecos (japonica WC), V20R(Indica isogenic restorer line), M202 (Japonica USA variety), Dular (Indica WC variety), GZ5310-20-3-3 (Japonica), Norin PL9 (Japonica WC) and Giza 178 (Indica / Japonica variety used as restorer for cytoplasmic male sterile lines) were used as pollen parents (testers) ( Table 1). The $F_{1}$ hybrid combinations along with their respective parents were grown in a randomized complet block design with three replications at Rice Research and Training Center (RRTC), Sakha, Kafr El-Sheikh, Egypt, in 2006and 2007 summer seasons. Thirty day -old seedlings were transplanted with one seedling hill-1 adopting a spacing of 20 $\mathrm{cm}$ between plants. Each test entry consisted of 3 rows of $5 \mathrm{~m}$ length.

Table(1): PTGMS male sterile and pollen parents lines used for the experiment.

\begin{tabular}{|l|c|c|}
\hline Genotype & Sterility source & Origin \\
\hline PTGMS-1 & Photoperiod thermo sensetive genic male sterile. & China-Egypt \\
\hline PTGMS-2 & Photoperiod thermo sensetive genic male sterile. & China-Egypt \\
\hline PTGMS-4 & Photoperiod thermo sensetive genic male sterile. & China-Egypt \\
\hline PTGMS-5 & Photoperiod thermo sensetive genic male sterile. & China-Egypt \\
\hline PTGMS-7 & Photoperiod thermo sensetive genic male sterile. & China-Egypt \\
\hline PTGMS-14 & Photoperiod thermo sensetive genic male sterile. & China-Egypt \\
\hline Pecos & Tester Japonica (WC) & Japan \\
\hline V20R & Tester Indica & China \\
\hline M202 & Tester japonica & USA \\
\hline Dular & Tester Indica (WC) & India \\
\hline GZ5310-20-3-3 & Tester Japonica & Egypt \\
\hline Norin PL9 & Tester Japonica (WC) & Japan \\
\hline Giza 178 & Indica / Japonica & China \\
\hline
\end{tabular}

Observation were recorded on five plants plot $^{-1}$ taken at random from each entry replication for plant height, panicle length, panicles plant ${ }^{-1}$, filled grains panicle ${ }^{-1}$, spikelets panicle ${ }^{-1}$, spikelet fertility and 100-grain weight. Ten plants from the middle row of three guarded rows were harvested from each entry in each replication to determine grain yield $\left(\mathrm{g} / \mathrm{plant}^{-1}\right)$.

Standard heterosis ( over the best inbred variety, Giza 178) was calculated for the studied characters while combining ability analysis was carried out as suggested by Kempthorne model (1957) for random lines 
representing certain population. The present represent selected set of males and females.

\section{RESULTS AND DISCUSSION}

Mean performance of 13 parental lines (six PTGMS lines as females and seven pollen parents as testers ) and their 42 hybrid genotype of line $x$ tester for the nine studied characters are presented in Table (2). The mean performance of the studied characters varied from one combination to another. With respect to plant height, the most desirable mean values towards short stature ( less than $110 \mathrm{~cm}$ ) were found in 21 hybrid combinations. Complete to over dominance was observed in most hybrids towards the taller parents for plant height, longest panicle, higher productive tillers plant $^{-1}$, heavier panicle weight, highest spikelets panicle ${ }^{-1}$ and heavier 100 -grain weight. However, some of the hybrids showed the same dominance effects towards the higher filled grains panicle ${ }^{-1}$, higher rate of spikelet fertility $\%$ and higher grain yield plant $^{-1}$. on the other hand, some hybrid combinations exhibited dominance effect towards the lower parents, Viz., for plant height ( no hybrids)for panicle length ( one hybrid), for productive tillers ( four hybrids), for spikelet fertility \%( 33 hybrids), for $100-$ grain weight ( one hybrid) and for grain yield plant ${ }^{-1}$ ( no hybrids). However, the rest of the hybrid combinations showed intermediate mean values between the parents involved for all studied characters.

The highest mean values of grain yield plant ${ }^{-1}(\mathrm{~g})$ were obtained by the hybrid combinations PTGMS-5 / Dular, PTGMS-14 / Norin PL9, PTGMS-2 / Dular, PTGMS-7 / Dular, PTGMS-14 / Giza 178 and PTGMS-5 / Giza 178 with values of $67.69 \mathrm{~g}, 66.56 \mathrm{~g}, 65.21 \mathrm{~g}, 65.08 \mathrm{~g}, 64.36 \mathrm{~g}$ and $63.49 \mathrm{~g}$, respectively. The lowest values were estimated for the hybrids PTGMS-1 / Norin PL9 (23.9g) and PTGMS-7 / V20R (20.54g). the parental lines Giza 178, M202, Gz5310-20-3-3 and Norin PL9, manifested highest mean performance of $46.6,43.31,40.29$ and 40.20 , respectively.

\section{Standard heterosis:}

The standard heterosis is especially important because the hybrid to be released is expected to outperform the existing superior local variety or hybrid. The data for standard heterosis in 42 hybrid combinations are presented in Table 3. Evaluation based on the standard heterosis revealed that three hybrids recorded significant negative standard heterosis for short stature plant ${ }^{-1}$. 31 hybrid combinations recorded significant positive standard heterosis for panicle length, 3 hybrids for productive tillers plant ${ }^{-1}, 12$ hybrids for panicle weight, 10 hybrids for filled grains panicle ${ }^{-1}, 15$ hybrids for spikelets panicle ${ }^{-1}, 10$ hybrids for spikelets fertility \%, all 42 hybrids for 100 grain weight and 22 hybrids for grain yield plant ${ }^{-1}$. The outstanding hybrid combinations for grain yield plant ${ }^{-1}$ were PTGMS-5 / Dular (45.26\%) with significant standard heterosis for panicle length (28.22\%), productive tillers plant $^{-1}$, panicle weight $(26.93 \%)$, filled grains panicle ${ }^{-1}(25.89 \%)$, spikelets panicle $^{-1}(14.19 \%)$, spikelet fertility $(10.20 \%)$ and 100-grain (35.62\%). 
El-Diasty, Z. M. et al.

T2 
J. Agric. Sci. Mansoura Univ., 33(5), May, 2008

T2c

3395 


\section{El-Diasty, Z. M. et al.}

T3

3396 
In the mean time, PTGMS-14 /Norin PL9 gave significant desirable SH\% grain yield plant $^{-1}(42.83 \%)$ with significant heterosis for panicle length (34.99\%), panicle weight $(47.63 \%)$, filled grain panicle ${ }^{-1}(20.45 \%)$, spikelets panicle $^{-1}(13.69 \%)$, spikelets fertility\% (5.91\%) and 100-grain weight (21.46\%). PTGMS-2 / Dular (39.94\%) with significant SH\% for panicle length $(24.60 \%)$, panicle weight $(21.94 \%)$, filled grains panicle ${ }^{-1}(3.76 \%)$, spikelet fertility\% (8.93\%), 100-grain weight (37.44\%). Also, PTGMS-7 / Dular $(39.66 \%)$ with significant $\mathrm{SH} \%$ for panicle length $(30.47 \%)$, panicle weight $(38.40 \%)$, filled grains panicle ${ }^{-1}(14.89 \%)$, spikelets panicle ${ }^{-1}(5.19 \%)$, spikelet fertility\% (9.16\%) and 100-grain weight (37.44\%). PTGMS-14 / Giza 178 (38.11\%) gave significant SH\% for panicle length $(13.77 \%)$, filled grains panicle ${ }^{-1}(11.59 \%)$, spikelets panicle ${ }^{-1}(10.48 \%)$ and 100-grain weight (11.87\%). However, PTGMS-5 / Giza 178 (36.24\%) gave significant standard heterosis for panicle length (17.83\%), filled grain panicle $(7.16 \%)$, spikelets panicle $^{-1}(15.59 \%)$ and 100-grain weight (12.79\%). Singh et al. (1980), Kim and Rutger (1988), Devaraj and Nadarajan (1996), Mishra and Pandey (1998), El-Mowafi (2001), Attia (2003), El-Refai (2002) and El-Mowafi (2005) also observed negative and positive standard heterosis for these traits.

\section{Analysis of Variance :}

Analysis of variance (table4) revealed highly significant difference among the 55 genotypes (42 hybrids ,6PTGMS lines and 7 testers ) tested for all nine studied characters. The parental lines and hybrids showe highly significant differences for all characters .Parents vs crosses mean square indicated that average heterosis was highly significant in all crosses for all studies characters under investigation except productive tillers plant ${ }^{-1}$.

The analysis of variance for combining ability given in table 4 revealed significant differences among PTGMS lines for productive tillers plant ${ }^{-1}$, filled grains panicle and spikelets panicle ${ }^{-1}$. On the other hand the testers exhibited significant differences for all the characters except panicle weight .The highly significant mean squares of lines $x$ testers for all characters ,indicated that they interacted and produced markedly different combining ability effects, and this might be due to the wide genetic diversity of lines and testers . The estimate of variance due to GCA was higher than that due to SCA for plant height and spikelets panicle ${ }^{-1}$ suggesting greater importance of additive genetic variance which is in agreement with the results of Sardane and Borthakur (1987),Lokaprakash et al .(1991),Attia(2001),El Refaee(2002),El-Mowafu and Abou-Shousha (2003) and El-Mowafi et $\mathrm{al}(2005)$.

In case of the rest of the characters preponderance of non-additive gene action was recorded by virtu of low GCA/SCA variance ratios in agreement to reports of Ram et al . (1991),Singh and Singh (1991) and ElMowafi (2001)

\section{General combining ability effects ( GCA):}

Significanmt differences of GCA effects were observed among the PTGMS lines for the studied characters (Table 4).The PTGMS-5 was best combiner for panicle length, productive tillers plant $^{-1}$, panicle weight, filled grains panicle $e^{-1}$, spikelets panicle ${ }^{-1}, 100$-grain weight and grain yield plant ${ }^{-1}$. 
PTGMS-14 was best combiner for panicle weight, filled grains panicle ${ }^{-1}$, spikelets panicle ${ }^{-1}$, spikelet fertility $\%$ and grain yield plant $^{-1}$. PTGMS-4 was best combiner for plant height, filled grains panicle ${ }^{-1}$, spikelets panicle ${ }^{-1}$ and spikelet fertility \%. However, PTGMS-7 was best combiner for spikelet fertility\% while, PTGMS-2 was best combiner for 100-grain weight.

Among the testers or male parents lines (Table 5 ), Giza 178 was the best general combiner for plant height, panicle length, productive tillers plant 1 , panicle weight, filled grains panicle ${ }^{-1}$, spikelets panicle ${ }^{-1}$, spikelet fertility and grain yield. The indica wide compatibility variety (WCV), Dular was best combiner for panicle length, productive tillers plant $^{-1}$, panicle weight, filled grains panicle ${ }^{-1}$, spikelet fertility\%, 100-grain weight and grain yield plant ${ }^{-1}$ and grain yield plant ${ }^{-1}$. The Japonica WC variety Pecos was best general combiner for panicle weight, filled grains panicle ${ }^{-1}$, spikelet fertility $\%$ and grain yield plant ${ }^{-1}$. According to the ranking numbers of the GCA, the data in Table (7) showed that the female lines (PTGMS), PTGMS-5, PTGMS-14 and PTGMS- 4 and also the male lines, Giza 178 Dular, Giza 178, M202 and Pecos were the best general combiners for the nine characters studied.

\section{Specific combining ability effects(SCA):}

The data of the SCA given in Table (6) revealed that there are some superior combinations that could be useful in the local breeding program to get good recombinants. With respect to plant height 16 hybrids showed significant effects in the desired direction for this trait. For panicle length, 11 hybrids had superior SCA effects, ??? for productive tillers plant ${ }^{-1}, 15$ for panicle weight, 17 for filled grains panicle ${ }^{-1}, 16$ for spikelets panicle ${ }^{-1}, 20$ for spikelet fertility \%, 15 for 100-grain weight and 23 hybrid combinations for grain yield plant $^{-1}$ were superior in SCA effects for panicle length, panicle weight, filled grains panicle, spikelets panicle ${ }^{-1}$, spikelet fertility $\%$ and grain yield plant ${ }^{-1}$. It was followed by PTGMS-1 / Dular for panicle length, panicle weight, filled grains panicl ${ }^{-1}$, spikelets panicle ${ }^{-1}$, spikelet fertility\%, 100-grain weight and grain yield plant $^{-1}$, then PTGMS-2 / Dular for panicle length, panicle weight, filled grains panicle ${ }^{-1}$, spikelets panicle ${ }^{-1}$, spikelet fertility $\%$

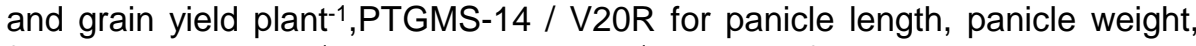
filled grains panicle ${ }^{-1}$, spikelets panicle ${ }^{-1}$, spikelet fertility $\%$ and grain yield plant $^{-1}$.

\section{Genetic parameters:}

The estimates of genetic parameters for the nine studied characters (Table 9) revealed that the additive variance $\left(\sigma^{2} A\right)$ and relative importance of GCA\% for plant height and spikelets panicle ${ }^{-1}$ were graeter than dominance variance $\left(\sigma^{2} D\right)$ and relative importance of SCA, respectively for these characters. The results indicate that the former character were largely governed by additive gene action. The importance of the additive gene action for the inheritance of these characters was in agreement with the finding of Lakaprakash et al. (1991), Sharma and Koranne (1995), El-Mowafi (2001), Attia (2001), El-Refae (2002), El-Mowafi and Abou shosha (2003) and ElMowafi et al.(2005). 
J. Agric. Sci. Mansoura Univ., 33(5), May, 2008

$\mathrm{T} 4-5-6$

3399 


\section{El-Diasty, Z. M. et al.}

T7 
J. Agric. Sci. Mansoura Univ., 33(5), May, 2008

T8

3401 


\section{El-Diasty, Z. M. et al.}

T9 
On the other hand, high estimates of dominance genetic variance and its relative magnitude of SCA\% were found to be more than those of the characters, such as panicle length, productive tillers plant ${ }^{-1}$, panicle weight, filled grains panicle ${ }^{-1}$, spikelet fertility\%, 100-grain weight and grain yield plant $^{-1}$. These results indicate that dominance variance played a major role in the inheritance of these characters which are in general agreement with the results reported by Ramalingram et al.(1997) and El-Mowafi et al.(2005) .

\section{REFERENCES}

Alam,-M-F; Khan,-M-R; Nuruzzaman,-M; Parvez,-S; Swaraz,-A-M; Alam,-I; Ahsan,-N. Genetic basis of heterosis and inbreeding depression in rice (Oryza sativa L.). Journal-of-Zhejiang-University-Science. 2004; 5(4): 406-411.

Attia, K.A. (2001). Evaluation and RAPD analysis of photo-thermosensitive genetic male sterile lines in Indica rice (Oryza sativa L.). M.Sc. Thesis, Institute of Genetics and Plant Breeding. College of Agric. and Biotechnol., Zhejang Univ., Hangzhou, P.R. China.

Devaraj, M. and N. Nadarajan (1996). Evaluation of rice hybrids. Oryza,33:230-235.

El-Mowafi, H.F. (2001a) Study on heterosis in hybrid rice under Egyptian conditions. Egypt. J. Appl. Sci., 16(2); 52-63.

El-Mowafi, H.F. (2001b). Combining ability analysis for some cytoplasmic male sterile and restorer rice lines under Egyptian conditions. Egypt. J. Appl. Sci., 16(2): 25-51.

El-Mowafi, H.F. and A.A. Abou-Shousha (2003). Combining ability and heterosis analysis of diverse CMS lines in hybrid rice. J. Agric. Res. Tanta Univ., 29(1): 106-127.

El-Refaee, Y.Z.E. (2002). Genetical and biochemical studies on heterosis and combining ability in rice. M.Sc. Thesis, Fac. Agric., Tanta Univ., Kafr ElSheikh, Egypt.

Kempthorne, O. (1957). An Introduction to Genetic Statistics. John Wiley and Sons Inc., New York, 458-471.

Kim, C.H. and J.N. Rutger (1988). Heterosis in rice. In "Hybrid Rice." Pp:3954. Int. Rice Res. Inst. Manila, Philippines.

Lokaprakash, R.; G. Shivashankar; M. Mahaderappa; B.T. Shankaregowda and R.S. Kulkami (1991). Combining ability for yield and its components in rice. Oryza 28(3): 319-322 (Cited From Rice Abstracts. 1995 Vol. 18, No. 1).

Mishra, M. and M.P. Pandey (1998). Heterosis breeding in rice for irrigated sub-humid tropics in North India. Oryza, 35(1): 8-14.

Ram, T., Singh and R.M. Singh (1991). Genetic analysis of yield and yield components in rice. Oryza 28:447-450.

Ramalingam, J.; N. Nadarajan; G. Vanniarajan and P. Rangaswamy (1997). Combining ability studies involving CMS lines in rice. Oryza, 34: 4-7.

Sardana, S. and D.N. Borthakur (1987). Combining ability for yield in rice. Oryza 24:14-18. 


\section{El-Diasty, Z. M. et al.}

Sharma, R.K. and K.D. Koranne (1995). Line $x$ tester analysis for yield and yield components in Indica $x$ Japonica crosses of rice. Oryza, 32: 234238.

Singh, R. and A. Singh (1991). Combining ability for harvest index and other related characters in rice (Oryza sativa L.). Oryza. 28: 19-22.

Singh, S.P., R.P. Singh, R.R. Singh and R.V. Singh(1980). Heterosis in rice Oryza (17):109-113.

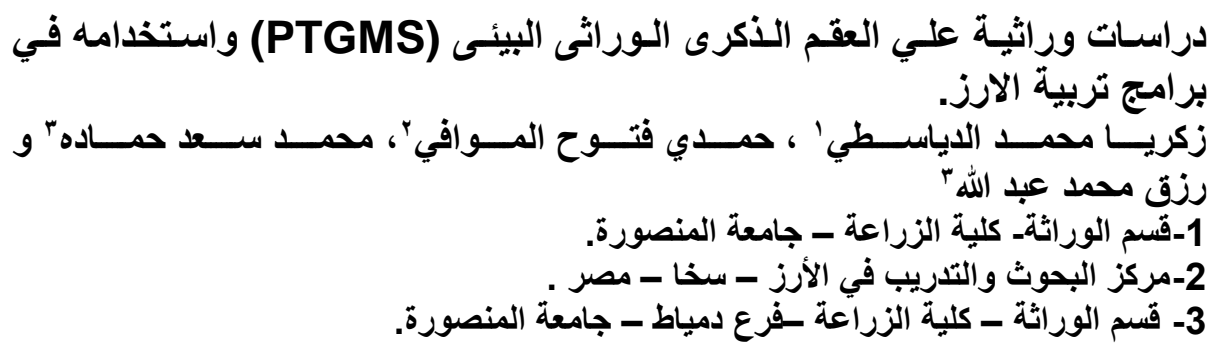

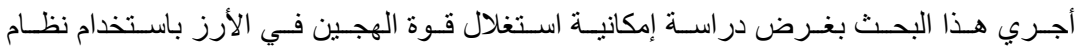

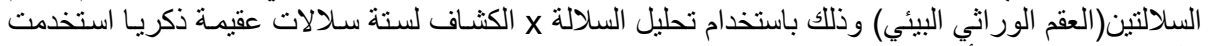

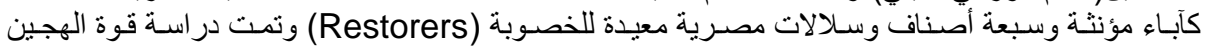

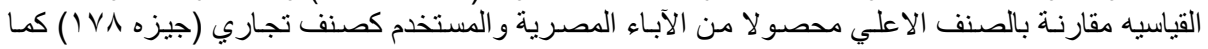

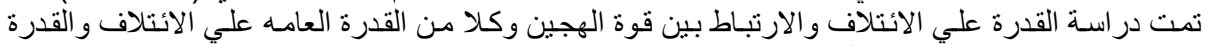

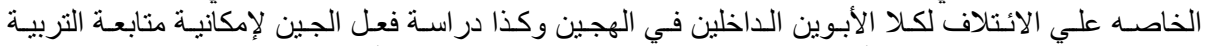

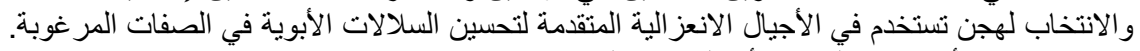

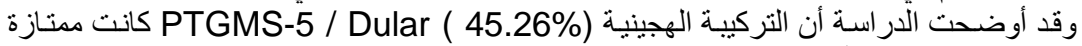

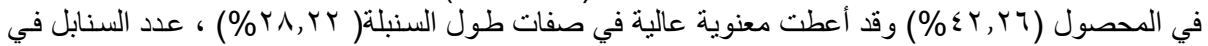

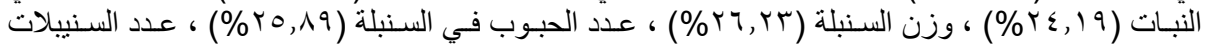

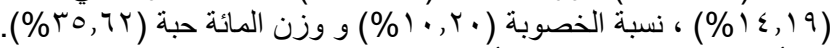

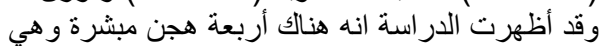

PTGMS-14 / Norin PL9, PTGMS-7 / Dular, PTGMS-14 / Giza 178 and PTGMS-5 / Giza 178.

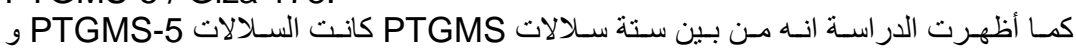

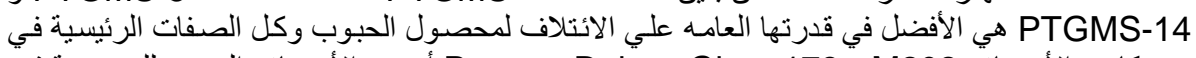

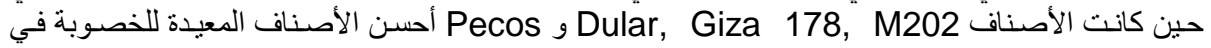
قدرتها العامة علي الائتلاف في صفة الدحصول ومعظم الصفات الرئيسية. 
J. Agric. Sci. Mansoura Univ., 33(5): 3391 - 3404, 2008

Table(2): Mean performance of parental lines (PTGMS and restorer lines ) and hybrid combinations for nine tudied characters.

\begin{tabular}{|c|c|c|c|c|c|c|c|c|c|}
\hline Genotype & $\begin{array}{c}\text { Plant height } \\
\text { (cm) }\end{array}$ & $\begin{array}{c}\text { Panicle } \\
\text { length } \\
\text { (cm) }\end{array}$ & $\begin{array}{l}\text { Productive } \\
\text { tillers plant }^{-1}\end{array}$ & $\begin{array}{c}\text { Panicle } \\
\text { weight } \\
\text { (g) }\end{array}$ & $\begin{array}{l}\text { Filled grain } \\
\text { panicle }^{-1}\end{array}$ & $\begin{array}{l}\text { Spikelets } \\
\text { panicle }^{-1}\end{array}$ & $\begin{array}{l}\text { Spikelet } \\
\text { fertility\% }\end{array}$ & $\begin{array}{c}\text { 100-grin } \\
\text { weight } \\
\text { (g) }\end{array}$ & $\begin{array}{l}\text { Grain yield } \\
\text { Plant }^{-1}\end{array}$ \\
\hline PTGMS (female): & & & & & & & & & \\
\hline PTGMS-1 & 54.50 & 18.05 & 25.3 & 1.12 & 22.58 & 96.5 & 90.45 & 2.26 & 19.24 \\
\hline PTGMS-2 & 56.00 & 18.20 & 28.5 & 1.17 & 24.05 & 89.3 & 92.30 & 2.39 & 22.05 \\
\hline PTGMS-4 & 55.50 & 18.35 & 25.5 & 1.28 & 26.60 & 91.9 & 95.03 & 2.29 & 22.01 \\
\hline PTGMS-5 & 55.25 & 19.28 & 27.8 & 0.96 & 28.45 & 99.7 & 92.83 & 2.35 & 18.45 \\
\hline PTGMS-7 & 59.75 & 19.45 & 29.5 & 1.05 & 29.23 & 118.6 & 90.03 & 2.30 & 19.41 \\
\hline $\begin{array}{l}\text { PTGMS-14 } \\
\text { Restorer lines(male): }\end{array}$ & 60.00 & 19.00 & 22.3 & 0.98 & 28.20 & 108.2 & 91.28 & 2.23 & 14.83 \\
\hline$\overline{P e c o s}$ & 97.25 & 18.48 & 17.3 & 3.13 & 127.83 & 136.7 & 93.45 & 2.47 & 36.57 \\
\hline V20R & 60.25 & 17.18 & 21.8 & 1.96 & 64.15 & 67.60 & 94.95 & 3.13 & 26.39 \\
\hline M202 & 96.75 & 19.68 & 22.8 & 3.79 & 150.05 & 164.6 & 91.15 & 2.66 & 43.31 \\
\hline Dular & 145.75 & 27.80 & 14.0 & 3.59 & 121.25 & 133.9 & 90.65 & 2.62 & 33.03 \\
\hline GZ5310 & 98.00 & 19.60 & 20.0 & 3.71 & 123.43 & 129.5 & 95.30 & 2.65 & 40.29 \\
\hline Norin PL9 & 92.00 & 21.42 & 19.0 & 3.60 & 112.65 & 123.7 & 91.10 & 2.31 & 40.20 \\
\hline $\begin{array}{l}\text { Giza } 178 \\
\text { Hybrid combinations: }\end{array}$ & 89.00 & 22.15 & 24.8 & 4.01 & 141.70 & 163.4 & 86.75 & 2.19 & 46.60 \\
\hline PGMS-1/Pecos & 115.15 & 22.80 & 19.8 & 4.14 & 132.13 & 147.3 & 89.25 & 2.62 & 54.84 \\
\hline N20R & 86.50 & 22.30 & 23.4 & 2.67 & 85.88 & 104.2 & 82.45 & 2.74 & 38.75 \\
\hline /M202 & 91.50 & 20.90 & 18.4 & 3.55 & 97.90 & 130.5 & 75.10 & 2.88 & 36.69 \\
\hline /Dular & 131.90 & 28.90 & 19.9 & 5.16 & 139.48 & 144.08 & 96.80 & 3.01 & 62.64 \\
\hline /GZ5310 & 108.25 & 25.50 & 21.1 & 3.32 & 74.15 & 151.95 & 48.75 & 2.58 & 25.60 \\
\hline /Norin PL9 & 113.23 & 23.80 & 19.4 & 2.92 & 78.48 & 138.68 & 56.58 & 2.79 & 23.90 \\
\hline / Giza 178 & 106.83 & 25.70 & 25.6 & 2.76 & 95.65 & 149.5 & 64.00 & 2.85 & 32.70 \\
\hline PGMS-2/Pecos & 82.00 & 23.40 & 19.1 & 4.16 & 130.40 & 144.15 & 90.45 & 2.60 & 53.76 \\
\hline N20R & 84.63 & 21.40 & 23.0 & 2.63 & 79.98 & 104.73 & 76.35 & 2.94 & 34.60 \\
\hline /M202 & 109.00 & 22.60 & 17.8 & 3.44 & 96.05 & 150.30 & 63.93 & 3.04 & 29.18 \\
\hline /Dular & 136.35 & 27.60 & 23.1 & 4.89 & 147.03 & 155.63 & 94.50 & 3.01 & 65.21 \\
\hline /GZ5310 & 107.00 & 24.00 & 22.6 & 3.46 & 90.78 & 177.20 & 51.23 & 3.03 & 28.74 \\
\hline /Norin PL9 & 112.75 & 23.60 & 20.8 & 3.44 & 66.65 & 133.63 & 49.85 & 3.03 & 25.98 \\
\hline / Giza 178 & 120.15 & 25.20 & 25.6 & 5.19 & 165.43 & 187.45 & 88.25 & 2.77 & 56.27 \\
\hline
\end{tabular}


El-Diasty, Z. M. et al.

Table(2):Cont. mean performance of parental lines (PTGMS and restorer lines ) and hybrid combinations for nine studied characters.

\begin{tabular}{|c|c|c|c|c|c|c|c|c|c|}
\hline Genotype & $\begin{array}{c}\text { Plant height } \\
\text { (cm) }\end{array}$ & $\begin{array}{c}\text { Panicle } \\
\text { length } \\
\text { (cm) }\end{array}$ & $\begin{array}{l}\text { Productive } \\
\text { tillers plant }^{-1}\end{array}$ & $\begin{array}{c}\text { Panicle } \\
\text { weight } \\
\text { (g) }\end{array}$ & $\begin{array}{l}\text { Filled grain } \\
\text { panicle }^{-1}\end{array}$ & $\begin{array}{l}\text { Spikelets } \\
\text { panicle }^{-1}\end{array}$ & $\begin{array}{l}\text { Spikelet } \\
\text { fertility\% }\end{array}$ & $\begin{array}{c}\text { 100-grin } \\
\text { weight } \\
\text { (g) }\end{array}$ & $\begin{array}{l}\text { Grain yield } \\
\text { Plant }^{-1}\end{array}$ \\
\hline PGMS-4/Pecos & 114.23 & 24.40 & 18.7 & 4.24 & 136.50 & 147.83 & 92.35 & 2.86 & 55.00 \\
\hline N20R & 103.83 & 24.70 & 23.8 & 4.27 & 112.48 & 151.83 & 62.60 & 2.90 & 47.68 \\
\hline /M202 & 111.20 & 24.50 & 20.4 & 4.04 & 120.48 & 160.78 & 74.95 & 3.00 & 46.32 \\
\hline /Dular & 119.10 & 23.90 & 27.3 & 2.45 & 88.23 & 127.15 & 69.38 & 2.53 & 34.70 \\
\hline /GZ5310 & 108.00 & 23.70 & 25.5 & 2.21 & 148.43 & 181.60 & 81.73 & 2.64 & 38.22 \\
\hline /Norin PL9 & 109.25 & 23.70 & 22.4 & 4.05 & 135.25 & 164.45 & 82.25 & 2.75 & 53.67 \\
\hline / Giza 178 & 97.25 & 24.40 & 25.2 & 4.46 & 183.08 & 190.53 & 96.10 & 2.47 & 62.06 \\
\hline PGMS-5/Pecos & 121.85 & 24.20 & 23.6 & 4.61 & 133.08 & 175.88 & 75.68 & 2.75 & 55.53 \\
\hline N20R & 85.45 & 21.40 & 23.2 & 3.67 & 128.90 & 155.45 & 82.93 & 2.85 & 53.01 \\
\hline /M202 & 103.58 & 23.70 & 24.7 & 3.59 & 125.50 & 179.15 & 70.05 & 3.04 & 50.94 \\
\hline /Dular & 131.83 & 28.40 & 20.8 & 5.09 & 178.38 & 186.58 & 95.60 & 2.97 & 67.69 \\
\hline /GZ5310 & 111.05 & 26.80 & 24.4 & 2.96 & 90.50 & 189.50 & 47.75 & 3.03 & 25.79 \\
\hline /Norin PL9 & 119.48 & 26.60 & 23.7 & 4.50 & 135.58 & 176.73 & 76.70 & 3.02 & 59.79 \\
\hline / Giza 178 & 103.08 & 26.10 & 26.2 & 4.25 & 151.85 & 188.88 & 80.40 & 2.47 & 63.49 \\
\hline PGMS-7/Pecos & 122.00 & 25.15 & 23.4 & 5.10 & 117.10 & 167.75 & 69.85 & 2.81 & 62.40 \\
\hline N20R & 88.00 & 20.40 & 22.7 & 1.50 & 99.95 & 124.13 & 80.53 & 3.16 & 20.54 \\
\hline /M202 & 108.13 & 20.35 & 23.2 & 3.86 & 127.80 & 162.78 & 78.50 & 2.90 & 52.64 \\
\hline /Dular & 134.6 & 28.90 & 23.4 & 5.55 & 162.80 & 171.88 & 94.70 & 3.01 & 65.08 \\
\hline /GZ5310 & 107.55 & 26.00 & 22.5 & 3.52 & 102.10 & 143.90 & 70.98 & 2.65 & 40.12 \\
\hline /Norin PL9 & 111.65 & 22.70 & 21.4 & 3.55 & 101.28 & 135.18 & 74.93 & 2.73 & 40.36 \\
\hline / Giza 178 & 106.70 & 25.90 & 24.7 & 4.14 & 136.18 & 167.75 & 81.18 & 2.38 & 55.71 \\
\hline PGMS-14/Pecos & 107.08 & 25.70 & 21.2 & 4.37 & 112.33 & 144.03 & 78.00 & 2.59 & 51.19 \\
\hline N20R & 110.15 & 24.20 & 19.6 & 4.88 & 143.98 & 162.28 & 88.73 & 2.79 & 55.11 \\
\hline /M202 & 115.15 & 24.50 & 24.4 & 4.88 & 110.15 & 185.60 & 59.35 & 2.75 & 41.31 \\
\hline /Dular & 123.3 & 18.50 & 20.8 & 3.22 & 160.50 & 168.68 & 95.15 & 2.06 & 47.66 \\
\hline /GZ5310 & 106.58 & 20.60 & 21.1 & 2.85 & 132.95 & 176.10 & 75.50 & 3.02 & 33.96 \\
\hline /Norin PL9 & 131.00 & 29.90 & 23.9 & 5.92 & 170.68 & 185.78 & 91.88 & 2.66 & 66.56 \\
\hline / Giza 178 & 110.38 & 25.20 & 25.2 & 4.18 & 158.13 & 180.53 & 87.60 & 2.45 & 64.36 \\
\hline
\end{tabular}


J. Agric. Sci. Mansoura Univ., 33(5), May, 2008

Table 3. Standard heterosis for nine characters of 42 hybrid combinations.

\begin{tabular}{|c|c|c|c|c|c|c|c|c|c|}
\hline Hybrid combinations & $\begin{array}{l}\text { Plant height } \\
\text { (cm) }\end{array}$ & \begin{tabular}{|c|} 
Panicle \\
length $(\mathrm{cm})$
\end{tabular} & $\begin{array}{c}\text { Productive } \\
\text { tillers plant }^{-1}\end{array}$ & $\begin{array}{c}\text { Panicle } \\
\text { weight (g) }\end{array}$ & \begin{tabular}{|c|}
$\begin{array}{c}\text { Filled grain } \\
\text { panicle }\end{array}$ \\
\end{tabular} & $\begin{array}{l}\text { Spikelets } \\
\text { panicle }^{-1}\end{array}$ & $\begin{array}{l}\text { Spikelet } \\
\text { fertility\% }\end{array}$ & $\begin{array}{c}\text { 100-grin } \\
\text { weight (g) }\end{array}$ & $\begin{array}{l}\text { Grain yield } \\
\text { Plant }^{-1}\end{array}$ \\
\hline PGMS-1/Pecos & $29.38^{* \star}$ & $2.93^{\text {ns }}$ & $-20.16^{\star *}$ & $3.24^{\text {ns }}$ & $-6.78^{* *}$ & $-9.85^{\star \star}$ & $2.88^{*}$ & $19.63^{\star \star}$ & $17.68^{\star \star}$ \\
\hline NV2OR & $\begin{array}{l}-2.81^{\text {ns }} \\
2.81^{\text {ns }}\end{array}$ & $0.68^{\mathrm{ns}}$ & $\begin{array}{l}-5.65^{\mathrm{ns}} \\
-25.81^{* *}\end{array}$ & $\begin{array}{l}-33.42^{* *} \\
-1147^{* *}\end{array}$ & $\begin{array}{l}-3.94^{* *} \\
-3.09^{* *}\end{array}$ & $\begin{array}{l}-36.23^{\star *} \\
-20.13^{\star *}\end{array}$ & $-4.96^{\star *}$ & $25.11^{\star *}$ & $\begin{array}{l}-16.85^{\star *} \\
-21 ? 7^{\star *}\end{array}$ \\
\hline /Dive & $48.20^{* *}$ & $\begin{array}{l}-5.64 \\
30.47^{* *}\end{array}$ & $\begin{array}{l}-25.81 \\
-19.76^{\star *}\end{array}$ & $\begin{array}{l}-11.47^{* *} \\
28.68^{* *}\end{array}$ & $\begin{array}{l}-3.09^{n} \\
-1.57^{\text {ns }}\end{array}$ & $-11.82^{\star \star}$ & $\begin{array}{l}-11.43 \\
11.59^{* *}\end{array}$ & $37.44^{* *}$ & $34.42^{* *}$ \\
\hline GZ5310 & $21.63^{* *}$ & $15.12^{* *}$ & $-14.92^{\star *}$ & $-17.21^{\star *}$ & $-47.67^{* *}$ & $-7.01^{* *}$ & $-43.80^{* *}$ & $17.81^{\text {** }}$ & $-45.06^{* *}$ \\
\hline /Norin PL9 & $27.22^{* *}$ & $7.45^{\star *}$ & $-21.77^{\star *}$ & $-29.68^{* *}$ & $-44.62^{* *}$ & $-15.13^{* *}$ & $-34.78^{\star *}$ & $27.40^{* *}$ & $-48.71^{* *}$ \\
\hline / Giza 178 & $20.03^{\star *}$ & $16.03^{* *}$ & $0.81^{\text {ns }}$ & $-31.17^{\star *}$ & $-32.50^{* *}$ & $-8.51^{\star *}$ & $-26.22^{\star *}$ & $30.01^{* *}$ & $-29.83^{\star *}$ \\
\hline$-2 / \mathrm{Pecos}$ & $-7.87^{\star *}$ & $5.64^{*}$ & $-22.98^{\star *}$ & $-3.74^{\text {ns }}$ & $-7.97^{* \star}$ & $-11.78^{* *}$ & $4.27^{* *}$ & $18.72^{* *}$ & $15.36^{* *}$ \\
\hline N2OR & $-4.91^{\star *}$ & $-3.39^{\text {ns }}$ & $7.26^{\star}$ & $-34.41^{* *}$ & $-43.56^{\star *}$ & $-36.35^{\star *}$ & $-11.99^{\star \star}$ & $34.25^{\star \star}$ & $-25.75^{\star \star}$ \\
\hline /M202 & $22.47^{* *}$ & $2.03^{\mathrm{ns}}$ & $-28.2^{* *} 3$ & $-14.21^{\star *}$ & $-32.22^{* *}$ & $-8.02^{* \star}$ & $-26.31^{\star *}$ & $38.81^{\star *}$ & $-37.38^{* *}$ \\
\hline /Dular & $\begin{array}{l}53.20^{\star *} \\
20 ? 2^{\star *}\end{array}$ & $24.60^{* *}$ & $\begin{array}{l}-6.85^{*} \\
-8.87^{* *}\end{array}$ & $21.94^{* *}$ & $3.76^{\star \star}$ & $-4.76^{\star *}$ & $8.93^{\star *}$ & $37.44^{* *}$ & $39.94^{* *}$ \\
\hline /Norin PL9 & $26.69^{* *}$ & $\begin{array}{l}8.35 \\
6.55^{*}\end{array}$ & $\begin{array}{l}-16.13^{* *} \\
-x^{*}\end{array}$ & $\begin{array}{l}-13 . / 2^{\star x} \\
-14.21^{* *}\end{array}$ & $\begin{array}{l}-35.94^{\star *} \\
-52.96^{* *}\end{array}$ & $\begin{array}{l}8.45^{* *} \\
-18.22^{* *}\end{array}$ & $\begin{array}{l}-40.95^{\star \star} \\
-42.54^{\star \star}\end{array}$ & $\begin{array}{l}38.36^{\star \star *} \\
38.36^{\star *}\end{array}$ & $\begin{array}{l}-38.33^{* \star} \\
-44.25^{\star *}\end{array}$ \\
\hline Giza 178 & $35.00^{* *}$ & $13.77^{\star *}$ & $0.31^{\text {ns }}$ & $-45.39^{\star \star}$ & $16.75^{\star *}$ & $14.44^{\star \star}$ & $1.73^{\mathrm{ns}}$ & $26.48^{\star *}$ & $20.75^{\star *}$ \\
\hline PGMS-4/Pecos & $28.35^{\star \star}$ & $10.16^{* *}$ & $-24.60^{* *}$ & $5.74^{\mathrm{ns}}$ & $-3.67^{\star *}$ & $-9.53^{* *}$ & $6.46^{* *}$ & $30.59^{* *}$ & $18.03^{\star *}$ \\
\hline N20R & $16.67^{* *}$ & $11.51^{* *}$ & $-4.03^{\text {ns }}$ & $6.48^{\text {ns }}$ & $-20.62^{* *}$ & $-7.08^{\star *}$ & $-27.84^{\star \star}$ & $32.42^{\star *}$ & $2.32^{\text {ns }}$ \\
\hline /M202 & $24.94^{\star *}$ & $10.61^{* *}$ & $-17.74^{\star \star}$ & $0.75^{\mathrm{ns}}$ & $-14.98^{\star \star}$ & $-1.60^{\mathrm{ns}}$ & $-13.60^{\star \star}$ & $36.99^{\star \star}$ & $-0.60^{\text {ns }}$ \\
\hline /Dular & $33.82^{* *}$ & $7.90^{* \star}$ & $10.08^{\star *}$ & $-38.90^{* *}$ & $-37.73^{\star *}$ & $-22.18^{\star *}$ & $-20.02^{\star *}$ & $15.53^{\star \star}$ & $.25 .54^{\star *}$ \\
\hline /GZ5310 & $21.35^{\star \star}$ & $7.00^{\star *}$ & $2.82^{\text {ns }}$ & $-44.89^{\star *}$ & $4.75^{\star \star}$ & $11.14^{\star *}$ & $-5.84^{\star *}$ & $20.55^{\star \star}$ & $-17.98^{* *}$ \\
\hline /Norin PL9 & $22.75^{\star *}$ & $7.00^{* \star}$ & $-9.68^{\star *}$ & $1.00^{\mathrm{ns}}$ & $-4.55^{\star *}$ & $0.64^{\text {ns }}$ & $-5.19^{\star *}$ & $25.57^{\star *}$ & $15.17^{\star *}$ \\
\hline / Giza 178 & $9.27^{\star \star}$ & $10.16^{* *}$ & $1.61^{\mathrm{ns}}$ & $11.22^{\star *}$ & $29.21^{* *}$ & $16.60^{* *}$ & $10.78^{\star *}$ & $12.79^{* *}$ & $33.18^{\star *}$ \\
\hline PGMS-5/Pecos & $36.91^{\star *}$ & $9.26^{\star \star}$ & $-4.84^{\mathrm{ns}}$ & $14.96^{\star \star}$ & $-6.08^{\star \star}$ & $7.64^{\star *}$ & $-12.76^{\star \star}$ & $25.57^{\star \star}$ & $19.16^{* \star}$ \\
\hline N20R & $3.99^{*}$ & $-3.39^{\text {ns }}$ & $-6.45^{\text {ns }}$ & $-8.48^{*}$ & $-9.03^{* *}$ & $-4.87^{\star *}$ & $-4.40^{* *}$ & $30.01^{* *}$ & $13.76^{\star \star}$ \\
\hline /M202 & $16.38^{* *}$ & $7.00^{\star *}$ & $-0.40^{\text {ns }}$ & $-10.47^{\star *}$ & $-11.43^{* *}$ & $9.64^{\star *}$ & $-9.25^{\star *}$ & $38.81^{\star *}$ & $9.31^{*}$ \\
\hline /Dular & $\begin{array}{l}48.12^{\star *} \\
24.78^{\star *}\end{array}$ & $\begin{array}{l}28.22^{* *} \\
20.99^{* *}\end{array}$ & $\begin{array}{l}24.19^{* *} \\
-161^{\text {ns }}\end{array}$ & $\begin{array}{l}26.93^{\star *} \\
-2618^{* *}\end{array}$ & $25.89^{* *}$ & $\begin{array}{l}14.19^{* *} \\
15.97^{* *}\end{array}$ & $\begin{array}{l}10.20^{\star *} \\
-44.96^{* *}\end{array}$ & $\begin{array}{l}35.62^{\star *} \\
38.36^{\star *}\end{array}$ & $\begin{array}{l}45.26^{* *} \\
-4466^{* *}\end{array}$ \\
\hline /Norin PL9 & $34.25^{* *}$ & $20.09^{* *}$ & $-4.44^{\text {ns }}$ & $12.22^{* *}$ & $-4.32^{* *}$ & $8.16^{* *}$ & $-11.59^{\star *}$ & $37.90^{\star *}$ & $28.30^{* *}$ \\
\hline / Giza 178 & $15.82^{\star *}$ & $17.83^{* *}$ & $5.65^{\mathrm{ns}}$ & $5.99^{\mathrm{ns}}$ & $7.16^{* *}$ & $15.59^{* *}$ & $-7.32^{\star \star}$ & $12.79^{\star \star}$ & $36.24^{\star \star}$ \\
\hline PGMS-7/Pecos & $37.08^{* *}$ & $13.54^{* *}$ & $-5.65^{\text {ns }}$ & $27.18^{\star \star}$ & $-17.36^{* *}$ & & $-19.40^{* *}$ & $28.31^{* *}$ & $33.91^{\star *}$ \\
\hline N2OR & $-1.12^{\text {ns }}$ & $-7.90^{\star *}$ & $017 *$ & $-62.59^{* *}$ & $-29.46^{* *}$ & $-24.03^{* *}$ & $-7.17^{\star \star}$ & $44.29^{* *}$ & $-55.92^{* *}$ \\
\hline /M202 & $21.49^{* *}$ & $-8.13^{* *}$ & & & $-9.81^{* *}$ & $-0.38^{\text {ns }}$ & $-9.51^{* *}$ & $32.42^{* *}$ & $12.96^{\star *}$ \\
\hline /Dular & $51.24^{* *}$ & $30.47^{* *}$ & $-5.65^{\mathrm{ns}}$ & $38.40^{* *}$ & $14.89^{* *}$ & $5.19^{* *}$ & $9.16^{* *}$ & $37.44^{\star *}$ & $39.66^{\star *}$ \\
\hline /GZ5310 & $20.84^{* *}$ & $17.38^{* *}$ & $-9.27^{* *}$ & $-12.22^{\star *}$ & $-27.95^{\star *}$ & $-11.93^{* *}$ & $-18.18^{\star *}$ & $21.00^{* *}$ & $-13.91^{* *}$ \\
\hline Norin PL9 & $25.45^{* *}$ & $2.48^{\mathrm{ns}}$ & $-13.71^{* *}$ & $-11.47^{* *}$ & $-28.53^{\star \star}$ & $-17.27^{\star *}$ & $-13.63^{\star *}$ & $24.66^{* *}$ & $-13.39^{\star *}$ \\
\hline / Giza 178 & $19.89^{\star \star}$ & $16.93^{* *}$ & $-0.40^{\mathrm{ns}}$ & $3.24^{\text {ns }}$ & $-3.90^{* *}$ & $2.66^{\mathrm{ns}}$ & $-6.42^{* *}$ & $8.68^{\star *}$ & $19.55^{* *}$ \\
\hline PGMS-14/Pecos & $20.31^{* *}$ & $16.03^{* *}$ & $-14.52^{\star *}$ & $8.98^{*}$ & $-20.73^{* *}$ & $-11.85^{\star *}$ & $-10.09^{* *}$ & $18.26^{* *}$ & $9.85^{\star}$ \\
\hline N20R & $23.76^{* *}$ & $9.26^{* *}$ & $-20.97^{\star \star}$ & $21.70^{\star *}$ & $1.61^{\mathrm{ns}}$ & $-0.69^{\text {ns }}$ & $2.28^{\text {ns }}$ & $27.40^{* *}$ & $18.26^{\star *}$ \\
\hline /M202 & $29.38^{* *}$ & $10.61^{* *}$ & -1.6 & $21.70^{* *}$ & $-22.27^{\star *}$ & $13.59^{* *}$ & $-31.59^{* *}$ & $25.57^{* *}$ & $-11.35^{\star *}$ \\
\hline /Dular & $38.54^{* *}$ & $-16.48^{\star *}$ & $-16.13^{\star *}$ & $-19.70^{\star *}$ & $13.27^{\star *}$ & 3.23* & $9.68^{* *}$ & $39.73^{* *}$ & $2.27^{\mathrm{ns}}$ \\
\hline /GZ5310 & & $-7.00^{* *}$ & $-14.92^{* *}$ & $-28.93^{* *}$ & $-6.18^{* *}$ & $7.77^{\star *}$ & $-12.97^{\star *}$ & $37.90^{* *}$ & $-27.12^{* *}$ \\
\hline /Norin PL9 & ** & & & & & 9 ** & $5.91^{\star *}$ & $21.46^{\star \star}$ & $42.83^{\star *}$ \\
\hline / Giza 178 & $24.02^{* *}$ & $13.77^{* *}$ & $1.61^{\mathrm{ns}}$ & $4.24^{\mathrm{ns}}$ & $11.59^{* *}$ & $10.48^{* *}$ & $0.98^{\text {ns }}$ & $1187^{* *}$ & $38.11^{* *}$ \\
\hline LSD 5\% & & 1.17 & & 0.32 & 4.57 & 4.57 & 2.18 & 0.062 & 4.57 \\
\hline $1 \%$ & 3.83 & 1.53 & 5.17 & 0.42 & 6.00 & $\begin{array}{l}6.00 \\
6.00\end{array}$ & 2.87 & 0.081 & $\begin{array}{l}4.01 \\
6.00\end{array}$ \\
\hline
\end{tabular}


Table (4) : Mean square estimates of ordinary analysis for nine studied characters.

\begin{tabular}{|c|c|c|c|c|c|c|c|c|c|c|}
\hline Source of variation & d. f. & $\begin{array}{c}\text { Plant } \\
\text { height } \\
\text { (cm) }\end{array}$ & $\begin{array}{c}\text { Panicle } \\
\text { length } \\
\text { (cm) }\end{array}$ & $\begin{array}{c}\text { Productive } \\
\text { tillers plant }^{-1}\end{array}$ & $\begin{array}{c}\text { Panicle } \\
\text { weight } \\
\text { (g) }\end{array}$ & $\begin{array}{c}\text { Filled grain } \\
\text { panicle }^{-1}\end{array}$ & $\begin{array}{l}\text { Spikelets } \\
\text { panicle }^{-1}\end{array}$ & $\begin{array}{l}\text { Spikelet } \\
\text { fertility\% }\end{array}$ & $\begin{array}{c}\text { 100-grin } \\
\text { weight } \\
\text { (g) }\end{array}$ & $\begin{array}{c}\text { Grain yield } \\
\text { Plant }^{-1}\end{array}$ \\
\hline Replication & 3 & $4.380^{\text {ns }}$ & $0.550^{\text {ns }}$ & $1.920^{\mathrm{ns}}$ & $0.021^{\mathrm{ns}}$ & $17.345^{\mathrm{ns}}$ & $2.964^{\mathrm{ns}}$ & $4.526^{\mathrm{ns}}$ & $0.002^{\mathrm{ns}}$ & $2.160^{\mathrm{ns}}$ \\
\hline Genotypes & 54 & $1921.03^{* *}$ & $39.361^{\star *}$ & $39.622^{\star \star}$ & $6.094^{\star *}$ & $6780.813^{\star \star}$ & $3579.509^{\star *}$ & $1795.313^{\star \star}$ & $0.277^{\star \star}$ & $918.589^{\star \star}$ \\
\hline Parents & 12 & $3019.54^{* *}$ & $29.276^{\star *}$ & $83.548^{* *}$ & $6.695^{\star *}$ & $10983.728^{* *}$ & $3379.509^{* *}$ & $4630.077^{\star *}$ & $0.270^{\star \star}$ & $480.837^{\star *}$ \\
\hline Hybrids & 41 & $636.27^{*}$ & $24.144^{\star *}$ & $27.705^{\star \star}$ & $3.703^{\star *}$ & $3649.816^{* *}$ & $2039.611^{* *}$ & $774.137^{\star \star}$ & $0.160^{\star \star}$ & $764.266^{\star \star}$ \\
\hline Parents VS crosses & 1 & $41413.77^{* *}$ & $784.269^{* *}$ & $1.127^{\mathrm{ns}}$ & $96.893^{* *}$ & $84716.693^{* *}$ & $69891.141^{* *}$ & $9646.367^{* *}$ & $5.177^{\star \star}$ & $12498.830^{\star \star}$ \\
\hline Females (F) & 5 & $168.52^{\text {ns }}$ & $4.195^{\mathrm{ns}}$ & $56.038^{\star \star}$ & $2.420^{\mathrm{ns}}$ & $6891.963^{*}$ & $6247.557^{\star *}$ & $356.238^{\mathrm{ns}}$ & $0.134^{\mathrm{ns}}$ & $862.945^{\mathrm{ns}}$ \\
\hline Males (M) & 6 & $2999.69^{*}$ & $41.123^{*}$ & $59.012^{\star \star}$ & $6.957^{\mathrm{ns}}$ & $7615.014^{\star \star}$ & $3950.984^{\star *}$ & $2155.242^{\star \star}$ & $0.436^{\star \star}$ & $2105.522^{\star \star}$ \\
\hline Females $\times$ Males $(F \times M)$ & 30 & $241.55^{*}$ & $24.073^{\star \star}$ & $16.721^{\star \star}$ & $3.266^{\star *}$ & $2316.419^{\star \star}$ & $956.013^{\star \star}$ & $567.566^{\star \star}$ & $0.109^{\star \star}$ & $479.569^{\star \star}$ \\
\hline Error & 162 & 4.414 & 0.710 & 1.457 & 0.054 & 7.849 & 10.862 & 2.282 & 0.002 & 8.051 \\
\hline CV \% & & 2.04 & 3.62 & 5.29 & 6.57 & 2.49 & 2.21 & 2.05 & 1.44 & 6.61 \\
\hline
\end{tabular}

CV \%

\begin{tabular}{l|c|}
\hline 2.04 & 3.62 \\
\hline 01 levels respectively.
\end{tabular}

5.29

Table (5). Estimates of GCA effects of PTGMS lines for the nine characters.

\begin{tabular}{|c|c|c|c|c|c|c|c|c|c|}
\hline Tester & \begin{tabular}{|c|} 
Plant height \\
(cm)
\end{tabular} & $\begin{array}{l}\text { Panicle } \\
\text { length } \\
\text { (cm) }\end{array}$ & $\begin{array}{l}\text { Productive } \\
\text { tillers plant }^{-1}\end{array}$ & $\begin{array}{l}\text { Panicle } \\
\text { weight } \\
\text { (g) }\end{array}$ & $\begin{array}{c}\text { Filled grains } \\
\text { panicle }^{-1}\end{array}$ & $\begin{array}{l}\text { Spikelets } \\
\text { panicle }^{-1}\end{array}$ & $\begin{array}{l}\text { Spikelet } \\
\text { fertility\% }\end{array}$ & $\begin{array}{l}\text { 100-grin } \\
\text { weight } \\
\text { (g) }\end{array}$ & $\begin{array}{c}\text { Grain yield } \\
\text { Plant }^{-1}\end{array}$ \\
\hline PTGMS-1 & $-3.1845^{\star \star}$ & 0.0119 & $-1.6667^{\star *}$ & $-0.3944^{\star \star}$ & $-23.3512^{\star \star}$ & $-21.0714^{\star \star}$ & $-3.8631^{\star \star}$ & $-0.0295^{\star}$ & $-7.8527^{\star \star}$ \\
\hline PTGMS-2 & 0.1726 & -0.3095 & $-1.0238^{* *}$ & -0.0094 & $-12.3869^{\star \star}$ & $-8.7500^{\star \star}$ & $-3.7917^{\star *}$ & $0.1034^{\star \star}$ & $-5.1920^{\star \star}$ \\
\hline PTGMS-4 & $-1.7560^{* *}$ & -0.1667 & 0.4405 & $-0.2205^{\star *}$ & $8.0060^{* \star}$ & $3.1429^{\star *}$ & $2.6012^{* *}$ & $-0.0770^{\star *}$ & 1.0769 \\
\hline PTGMS-5 & 0.1726 & $0.7619^{\star *}$ & $2.3690^{\star *}$ & $0.1992^{\star *}$ & $11.6488^{\star \star}$ & $19.7500^{\star *}$ & $-1.6131^{\star \star}$ & $0.0620^{\star *}$ & $6.7023^{\star \star}$ \\
\hline PTGMS-7 & 0.5298 & -0.1310 & 0.3690 & -0.0087 & $-2.0298^{* *}$ & $-5.7857^{\star *}$ & $1.7083^{* *}$ & -0.0084 & 0.9698 \\
\hline PTGMS-14 & $4.0655^{\star *}$ & -0.1667 & -0.4881 & $0.4338^{\star *}$ & $18.1131^{* *}$ & $12.7143^{* *}$ & $4.9583^{* *}$ & $-0.0505^{\star *}$ & $4.2958^{\star \star}$ \\
\hline \begin{tabular}{|l|l} 
LSD $5 \%$ \\
\end{tabular} & 1.10 & 0.44 & 0.63 & 0.12 & 1.47 & 1.73 & 0.79 & & 1.49 \\
\hline $1 \%$ & 1.45 & 0.58 & 0.83 & 0.16 & 1.93 & 2.27 & 1.04 & 0.031 & 1.95 \\
\hline
\end{tabular}

$1 \%$

1.10

0.58

0.83

Table 6. Estimates of GCA effects of tester lines for the nine studied characters.

\begin{tabular}{|c|c|c|c|c|c|c|c|c|c|}
\hline Tester & $\begin{array}{l}\text { Plant height } \\
\text { (cm) }\end{array}$ & $\begin{array}{l}\text { Panicle } \\
\text { length } \\
\text { (cm) }\end{array}$ & $\begin{array}{l}\text { Productive } \\
\text { tillers plant }^{-1}\end{array}$ & $\begin{array}{c}\text { Panicle } \\
\text { weight } \\
\text { (g) }\end{array}$ & $\begin{array}{l}\text { Filled grains } \\
\text { panicle }^{-1}\end{array}$ & $\begin{array}{l}\text { Spikelets } \\
\text { panicle }^{-1}\end{array}$ & $\begin{array}{l}\text { Spikelet } \\
\text { fertility\% }\end{array}$ & $\begin{array}{c}\text { 100-grin } \\
\text { weight } \\
\text { (g) }\end{array}$ & $\begin{array}{l}\text { Grain yield } \\
\text { Plant }^{-1}\end{array}$ \\
\hline Pecos & $3.8274^{\star \star}$ & -0.0179 & $-1.7738^{\star \star}$ & $0.5398^{\star \star}$ & $3.7500^{\star *}$ & $-4.6905^{\star *}$ & $5.4167^{\star *}$ & $-0.1071^{\star \star}$ & $8.4200^{\star \star}$ \\
\hline V20R & $-17.7560^{\star *}$ & -2.0179 & -0.1488 & $-0.6252^{* *}$ & $-16.6667^{* *}$ & $-22.3571^{* *}$ & $1.6667^{\star *}$ & $0.0808^{\star *}$ & $-5.5413^{* *}$ \\
\hline M202 & $-4.2560^{\star *}$ & $-1.4762^{* *}$ & $-1.3155^{\star *}$ & -0.0039 & $-10.1667^{\star *}$ & 1.5179 & $-7.0000^{* *}$ & $0.1245^{\star *}$ & $-4.3071^{* *}$ \\
\hline Dular & $18.7857^{* *}$ & $1.5238^{* *}$ & $1.4345^{\star \star}$ & $0.4957^{* \star}$ & $22.9167^{* *}$ & -0.1071 & $13.7917^{\star *}$ & $0.1195^{\star *}$ & $10.0104^{\star *}$ \\
\hline GZ5310 & $-2.7143^{\star *}$ & 0.1905 & 0.0595 & $-0.8431^{* *}$ & $-16.6667^{\star *}$ & $10.8512^{\star *}$ & $-14.1250^{\star \star}$ & 0.0129 & $-15.0804^{\star *}$ \\
\hline Norin PL9 & $5.4940^{* *}$ & $0.7321^{* *}$ & $-0.8988^{* *}$ & $0.1677^{\star *}$ & $-8.3750^{* *}$ & $-3.4821^{\star *}$ & $-5.3333^{* *}$ & 0.0158 & $-2.1121^{\star *}$ \\
\hline Giza 178 & $-3.3810^{* *}$ & $1.0655^{\star \star}$ & $2.6429^{* *}$ & $0.2690^{* *}$ & $25.2083^{* *}$ & $18.2679^{\star *}$ & $5.5833^{\star *}$ & $-0.2463^{* *}$ & $8.6104^{\star *}$ \\
\hline LSD $5 \%$ & 1.189 & 0.48 & 0.683 & 0.131 & 1.585 & 1.867 & 0.821 & 0.0253 & 1.605 \\
\hline $1 \%$ & 1.562 & 0.63 & 0.898 & 0.172 & 2.083 & 2.451 & 1.079 & 0.0333 & 2.110 \\
\hline
\end{tabular}

*, ${ }^{* *}$ Significant at 0.05 and 0.01 levels, respectively. 
J. Agric. Sci. Mansoura Univ., 33(5), May, 2008

Table(8). Estimates of SCA effects of the hybrid combinations.

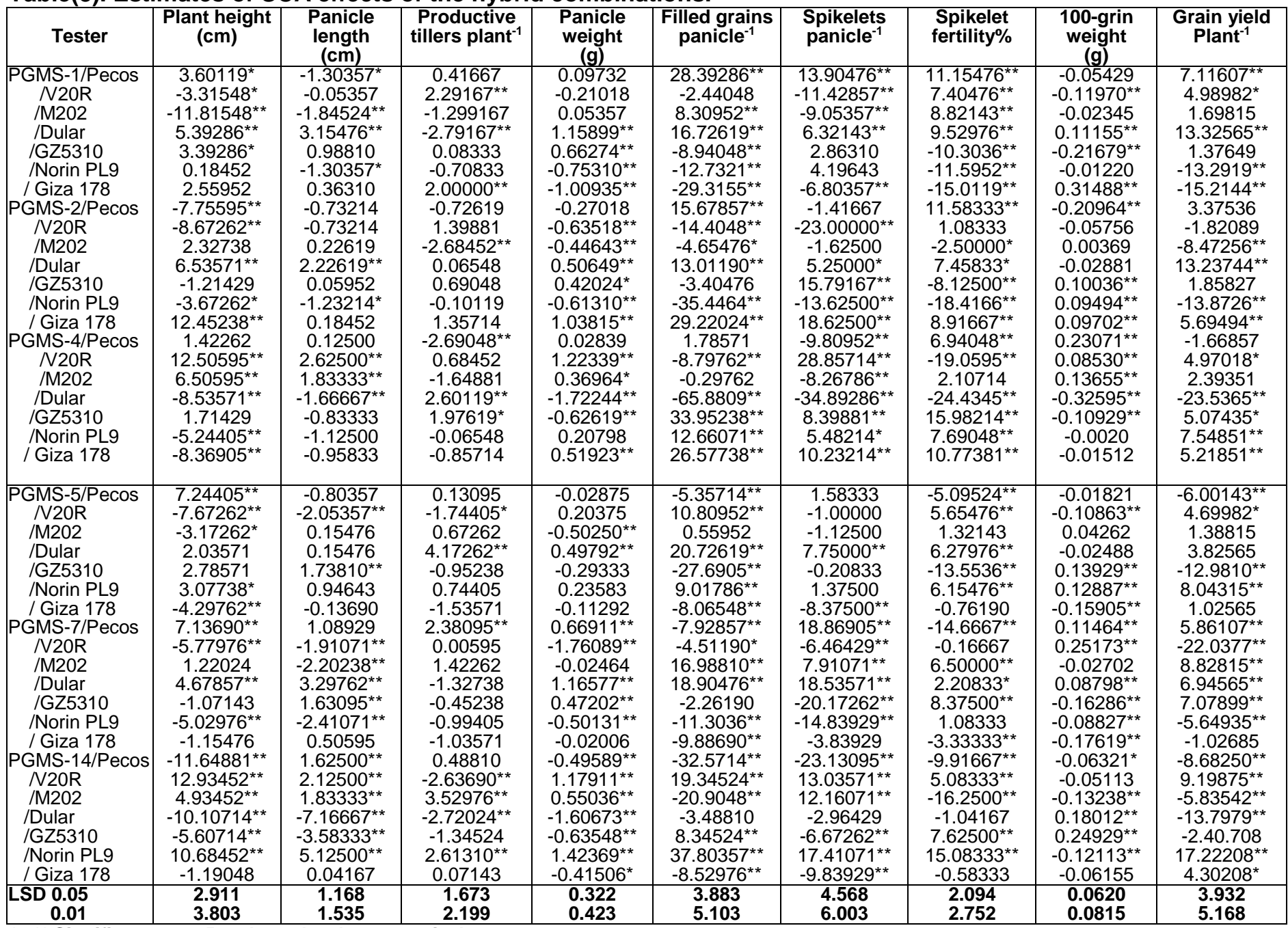

* ** Significant at 0.05 and 0.01 levels, respectively. 


\section{El-Diasty, Z. M. et al.}

Table 9. Genetic parameters for nine studied characters..

\begin{tabular}{|c|c|c|c|c|c|c|c|c|c|}
\hline Parameter & $\begin{array}{l}\text { Plant } \\
\text { height } \\
(\mathrm{cm})\end{array}$ & $\begin{array}{c}\text { Panicle } \\
\text { length } \\
(\mathrm{cm})\end{array}$ & $\begin{array}{c}\text { Productive } \\
\text { tillers plant }^{-1}\end{array}$ & $\begin{array}{c}\text { Panicle } \\
\text { weight } \\
(\mathrm{g})\end{array}$ & $\begin{array}{c}\text { Filled } \\
\text { grain } \\
\text { panicle }^{-1}\end{array}$ & \begin{tabular}{|l|} 
Spikelets \\
panicle $^{-1}$
\end{tabular} & $\begin{array}{r}\text { Spikelet } \\
\text { fertility\% }\end{array}$ & $\begin{array}{c}\text { 100-grin } \\
\text { weight } \\
(\mathrm{g})\end{array}$ & $\begin{array}{c}\text { Grain } \\
\text { yield } \\
\text { Plant }^{-1}\end{array}$ \\
\hline Additive variance $\left(\sigma^{2} \mathrm{~A}\right)$ & 112.31 & 0.0005 & 3.17 & 0.184 & 384.20 & 313.40 & 57.48 & 0.02 & 81.44 \\
\hline Dominant variance $\left(\sigma^{2} D\right)$ & 59.28 & 5.841 & 3.82 & 0.803 & 577.14 & 236.29 & 142.07 & 0.03 & 117.88 \\
\hline Environmental variance $\left(\sigma^{2} \mathrm{E}\right)$ & 4.41 & 0.710 & 1.46 & 0.054 & 7.85 & 10.86 & 2.48 & 0.002 & 8.05 \\
\hline Genotypic variance $\left(\sigma^{2} \mathrm{G}\right)$ & 176.01 & 5.841 & 6.98 & 0.987 & 961.34 & 549.69 & 199.59 & 0.04 & 199.32 \\
\hline Phenotypic variance $\left(\sigma^{2} P\right)$ & 171.60 & 6.551 & 8.44 & 1.041 & 969.19 & 560.55 & 202.02 & 0.04 & 207.37 \\
\hline Broad sense heritability $\left(h^{2} b\right) \%$ & 97.49 & 89.162 & 82.74 & 94.812 & 99.19 & 98.06 & 98.77 & 95.38 & 96.12 \\
\hline Narrow sense heritability $\left(h^{2} n\right) \%$ & 63.81 & 00.745 & 37.52 & 17.676 & 39.64 & 55.91 & 28.45 & 33.55 & 39.27 \\
\hline Relative importance of gca \%* & 65.45 & 00.008 & 45.35 & 18.643 & 39.96 & 57.01 & 28.80 & 35.18 & 10.86 \\
\hline Relative importance of sca \%** & 34.55 & 99.992 & 54.65 & 81.136 & 60.04 & 42.99 & 71.20 & 64.82 & 89.14 \\
\hline
\end{tabular}

* , ${ }^{*}$ Relative importance gca $=\sigma^{2} A / \sigma^{2}$ G x 100 and Relative importance sca $=\sigma^{2} D / \sigma^{2} G \times 100$, respectively. 
J. Agric. Sci. Mansoura Univ., 33(5), May, 2008

Table (7): Ranks of lines and testers for the nine studied characters.

\begin{tabular}{|c|c|c|c|c|c|c|c|c|c|c|c|}
\hline Genotype & $\begin{array}{l}\text { Plant } \\
\text { height } \\
\text { (cm) }\end{array}$ & $\begin{array}{c}\text { Panicle } \\
\text { length } \\
\text { (cm) }\end{array}$ & $\begin{array}{l}\text { Producti } \\
\text { ve tillers } \\
\text { plant }^{-1}\end{array}$ & $\begin{array}{c}\text { Panicle } \\
\text { weight } \\
\text { (g) }\end{array}$ & $\begin{array}{c}\text { Filled } \\
\text { grains } \\
\text { panicle }^{-1}\end{array}$ & $\begin{array}{l}\text { Spikelets } \\
\text { panicle }^{-1}\end{array}$ & $\begin{array}{c}\text { Spikelet } \\
\text { fertility } \\
\%\end{array}$ & $\begin{array}{c}\text { 100-grin } \\
\text { weight } \\
\text { (g) }\end{array}$ & $\begin{array}{c}\text { Grain } \\
\text { yield } \\
\text { Plant }^{-1}\end{array}$ & Total & Ranks \\
\hline \multicolumn{12}{|l|}{ PTGMS (female) } \\
\hline PTGMS-1 & 1 & 2 & 6 & 6 & 6 & 6 & 6 & 4 & 6 & 37 & 5 \\
\hline PTGMS-2 & 3 & 6 & 5 & 4 & 5 & 5 & 5 & 1 & 5 & 39 & 6 \\
\hline PTGMS-4 & 2 & 4 & 2 & 5 & 3 & 3 & 2 & 6 & 3 & 30 & 3 \\
\hline PTGMS-5 & 3 & 1 & 1 & 2 & 2 & 1 & 4 & 2 & 1 & 17 & 1 \\
\hline PTGMS-7 & 5 & 3 & 3 & 3 & 4 & 4 & 3 & 3 & 4 & 32 & 4 \\
\hline PTGMS-14 & 6 & 5 & 4 & 1 & 1 & 2 & 1 & 5 & 2 & 27 & 2 \\
\hline \multicolumn{12}{|l|}{ Restorer lines (male) } \\
\hline Pecos & 5 & 5 & 7 & 1 & 3 & 6 & 3 & 6 & 3 & 39 & 3 \\
\hline V20R & 1 & 7 & 4 & 6 & 6 & 7 & 4 & 3 & 6 & 44 & 5 \\
\hline M202 & 2 & 6 & 6 & 5 & 5 & 3 & 6 & 1 & 5 & 39 & 3 \\
\hline Dular & 7 & 1 & 2 & 2 & 2 & 4 & 1 & 2 & 1 & 20 & 1 \\
\hline GZ5310 & 4 & 4 & 3 & 7 & 6 & 2 & 7 & 5 & 7 & 45 & 6 \\
\hline Norin PL9 & 6 & 3 & 5 & 4 & 4 & 5 & 5 & 4 & 4 & 40 & 4 \\
\hline Giza 178 & 3 & 2 & 1 & 3 & 1 & 1 & 2 & 7 & 2 & 22 & 2 \\
\hline
\end{tabular}

